\title{
Relationship of Type II Diabetes Mellitus in Women with Polycystic Ovarian Syndrome: A Systematic Review
}

\author{
Aleena Batool $^{1} \quad$ Sayeda Khadija-tul-Sughra Marrium ${ }^{1} \quad$ Saira Islam $^{2} \quad$ Adeel kiyani $^{2}$ \\ Hasham Hafeez Hanjra $^{3}$ Dr.Humayun Azam ${ }^{3}$ \\ University Institute of Radiological Sciences and Medical Imaging Technology, Faculty of Allied Health \\ Sciences University of Lahore
}

\begin{abstract}
Objective: The purpose of my study was to determine the relationship of Type 2 diabetes mellitus in women with PCOS. Background: PCOS is a hormonal disorder affecting millions of women around the world. 1 in every 10 women have PCOS but many women go years before receiving the diagnosis. In fact it is estimated that $70 \%$ of PCOS cases have a root cause is insulin resistance, A major risk factor for Type 2 diabetes mellitus. More than half of women with PCOS develop type 2 diabetes. PCOS is linked with higher levels of circulating insulin, which is characteristic in type 2 diabetes. IR is the typical condition of subjects with T2D. Women with PCOS share with people with T2D the same impaired glucose pattern consisting of a prevalent disturbance of fasting blood glucose. Higher levels of IR stress the pancreatic beta cell function, resulting in earlier functional depletion of insulin secretion capacity and higher risk of developing T2D. In this Systematic review I wanted to determine the incidence of type 2 diabetes in women with PCOS. According to the studies that I reviewed women with PCOS have a markedly increased risk of developing type 2 diabetes then women without PCOS and there is a higher prevalence of PCOS in those women who have Type 2 diabetes mellitus rather than non-diabetic. Method:An electric database search was performed (google scholar, PubMed and science direct) without time limit until january 2020. All studies, fully available in English, assessing the incidence Type II Diabetes Mellitus in Women with PCOS. Study design: Systematic review. Results:I reviewed 22 articles and found that there were high risk of Diabetes Mellitus Type 2 in PCOS females then other one. Middle aged women with PCOS were having elevated risk of Type 2 Diabetes Mellitus, So therefore it propped up the needs of PCOS for routine screening of diabetes. The information or data from articles likewise gave furthur clinical bits of knowledge on the side of focusing on BMI (Body Mass Index), glucose (fasting glucose and after glucose ingestion) and in risk stratification and intervention for glucose homeostasis maintenance.
\end{abstract}

Keywords: Polycystic Ovarian Disease, Type II Diabetes Mellitus, Insulin Resistance

DOI: $10.7176 / \mathrm{JHMN} / 90-13$

Publication date: June $30^{\text {th }} 2021$

\section{Introduction}

Polycystic ovary syndrome (PCOS) and type 2 diabetes mellitus are both common conditions associated with insulin resistance and compensatory hyper insulinaemia. Polycystic ovary syndrome (PCOS) has, since its first description by Stein and Leventhal in 1935, become one of the commonest disorders in women, affecting 5 to $10 \%$ in the reproductive age-group ${ }^{1}$. This common medical condition has brought gynecologists, endocrinologists, cardiologists, pediatricians, and dermatologists together ${ }^{2}$.The disorder manifests as obesity, hirsutism, menstrual disturbances, acne vulgaris, male-pattern baldness, recurrent abortions, infertility, an-ovulation, and psychological and psychosexual morbidity ${ }^{3}$.It is an important cause of hirsutism and of infertility in our population ${ }^{4}$. With the advances and refinement in ultrasound technology, the presence of polycystic appearance of ovaries has increasingly been accepted as an essential part of the diagnosis of this syndrome and ultrasound suggestion of PCO has recently been introduced as one of the three diagnostic criteria of PCOS in Rotterdam consensus workshop $2003^{5}$.Using the most conservative criteria for transabdominal studies, such as presence of $\geq 10$ peripheral cysts per ovary, each $2-8 \mathrm{~mm}$, and thickened stroma, some authors have shown that as many as $21-23 \%$ of normal female population have $\mathrm{PCO}$ on ultrasound ${ }^{6}$.Some women with otherwise confirmed PCOS may have normal sized ovaries on ultrasound and normal sized ovaries without increased folicllarity don't preclude the diagnosis in proper clinical settings ${ }^{7}$.The pathophysiology of PCOS, one of the commonest endocrinopathies, is far from clear. A plethora of data point to insulin resistance (IR) and hyperinsulinemia as the central factor, a suggestion supported by clinical benefits of insulin sensitizers ${ }^{8}$. T2DM, which accounts for $90-95 \%$ of all diabetes mellitus, is a common metabolic disorder, characterized by a combination of IR and insulin secretory defects, occurring in varying proportions 9 . Both T2DM and PCOS are now considered to be two metabolically similar but phenotypically different expressions of the same syndromic continuum with IR being the common and pivotal link, which may be genetically determined ${ }^{10}$. Women with PCOS are also at increased risk for cardiovascular disease (CVD), given the high prevalence of the metabolic syndrome among them ${ }^{11}$. Many studies have revealed an increased prevalence of various abnormalities of glucose tolerance in women with PCOS. Polycystic ovary syndrome (PCOS) is the most frequent endocrine condition in women, but the diagnosis of PCOS is only possible during premenopausal 
years. The Rotterdam Criteria are used to establish the diagnosis of PCOS when other causes of the clinical phenotype are excluded. PCOS is characterized by insulin resistance and hyperinsulinemia and insulin resistance is closely associated with obesity in PCOS. The new guidelines for the management of women with PCOS, published in 2018, recommend that glycemic status should be assessed at time of diagnosis and thereafter every 1-3 years, depending on the considered risk, for early detection of IGT and/or T2DM ${ }^{12}$. It is important to study if development of T2DM can be predicted.

\section{Discussion}

According to the Office on Women's Health, around half of those women with PCOS develop either diabetes or prediabetes before the age of 40 .

Research from 2017 by Katrine Hass Rubin suggests that people with PCOS are four times more likely to develop type 2 diabetes than similar peers without the disorder and that diabetes is diagnosed four years earlier in women with PCOS compared to controls ${ }^{13}$.

A study by A. E. Joham: Around 8,000 females found that those with PCOS had a much higher risk of developing gestational or type 2 diabetes. The researchers note that this finding was independent of body mass ${ }^{14}$.

According to a review from 2016, various other studies have found a link between type 2 diabetes and PCOS.

In $2005 \mathrm{~A}$ prospective study by Abdul H.Zargar was done and objective of this srudy was Prevlence of Ultrasonography proved polycystic ovaries in North women with T2DM. One hundred and five reproductive age group women with diet and /or oral hypoglycemic treated T2DM were done. Sixty age-matched non-diabetic women served as controls. Ultrasonographic prevalence of PCO was higher in women with diabetes than in nondiabetic subjects $(61.0 \%$ vs. $36.7 \%, \mathrm{P}<0.003)$ whereas that of PCOS was $37.1 \%$ in diabetic subjects and $25 \%$ in non-diabetic controls $(\mathrm{P}>0.1)$. Diabetic women with PCO had diabetes of significantly longer duration than those without PCO $(4.19 \pm 2.0$ versus $2.9 \pm 1.6 \mathrm{yrs} ; \mathrm{p}<0.05)$. This study demonstrates a higher prevalence of PCO in women with T2DM as compared to non-diabetic subjects ${ }^{15}$.

In 2001 Jenifer J.conn published a case on cross sectional study and objective of this study was The prevalence of polycystic ovaries in women with type 2 diabetes mellitus. 49 women were presented of whom 38 $(76 \%)$ patients agreed to be studied. Eighty - two percent of women with type 2 diabetes mellitus had polycystic ovaries on ultrasound. Of these women, $52 \%$ had clinical evidence of cutaneous hyperandrogenism and/or menstrual disturbance. Women with type 2 diabetes mellitus have a higher prevalence of polycystic ovaries than that reported in the general population. Not all women with hyperinsulinaemia due to type 2 diabetes mellitus, however, develop PCO suggesting that hyper insulinaemia alone is not sufficient for the expression of this ovarian morphology ${ }^{16}$.

In 2002, Review of published literature was done by Ovalle F and the objective of this study was to review the definition and prevalence of two insulin resistance (IR)-associated phenotypes, polycystic ovary syndrome (PCOS) and type 2 diabetes mellitus, as well as the risk and nature of their simultaneous presentation. Insulin resistance affects between $10 \%$ and $25 \%$ of the general population. Two common disorders frequently associated with IR are PCOS, affecting 4\% to $6 \%$ of reproductive-aged women, and type 2 diabetes mellitus, which is observed in about $2 \%$ to $6 \%$ of similarly aged women. Overall, about $50 \%$ to $70 \%$ of women with PCOS and $80 \%$ to $100 \%$ of patients with type 2 diabetes mellitus have variable degrees of IR. Insulin resistance and its secondary hyperinsulinemia appear to underlie many of the endocrine features of PCOS in a large proportion of such patients. The risk of type 2 diabetes mellitus among PCOS patients is 5- to 10-fold higher than normal. In turn, the risk of PCOS among reproductive-aged type 2 diabetes mellitus patients appears to be similarly increased. It remains to be determined whether PCOS and type 2 diabetes mellitus represent no more than different clinical manifestations of the same IR syndrome, with their phenotypic differences due to the presence or absence of a coincidental genetic defect at the level of the ovary or pancreas, respectively, or representing the result of etiologically different subtypes of IR syndromes ${ }^{17}$.

In 2006, Monique Y done a study and objective of his study was know the Risk of T2DM and impaired fasting glucose among pcos subjects. follow-up of 8 years and Women with polycystic ovary syndrome (PCOS) often develop type 2 diabetes mellitus (T2DM). To determine the magnitude of risk of developing T2DM among women with PCOS, development of T2DM was prospectively assessed among women who had T2DM ( $\mathrm{n}=97)$ and controls $(\mathrm{n}=95)$ using Kaplan-Meier survival analysis and Cox proportional hazards modeling. The women with T2DM had higher baseline weight (body mass index, $\mathrm{P}<0.01$ ) and lower insulin sensitivity (homeostasis assessment model of insulin resistance, $\mathrm{P}<0.01$ ). The 8 -year incidence rate among cases and controls was $13.4 \%$ and $5.8 \%$, respectively (relative risk $=2.3$ ). Obese cases had a fivefold risk of T2DM developing $(\mathrm{P}<0.01)$ compared with age-adjusted obese controls, indicating significant interaction between PCOS and obesity to effect T2DM risk ${ }^{18}$.

Heather R. Peppard done a study and objective of this study was to determine the prevalence of PCOS among women with type 2 diabetes, He conducted a retrospective cross-sectional prevalence study. He reviewed the medical records of all women seen in the Diabetes Clinic of the Medical College of Virginia Hospitals between 
January 1995 through February 2000. 618 women with diabetes and identified 47 women eligible for study. Of the 47 women, 30 consented to an evaluation. Of the 30 women evaluated, 8 were identified as having PCOS resulting in a prevalence of $26.7 \%$. He concluded that PCOS occurs frequently among women with type 2 diabetes ${ }^{19}$

Panicha Chantrapanichkul in July 2019, done a retrospective cohort study and objective of his study was to investigate the prevalence of type 2 diabetes mellitus (T2DM) at the 5-year follow-up after polycystic ovarian syndrome (PCOS) diagnosis compared between lean and overweight/obese groups. This retrospective cohort study included who 400 prediabetes PCOS women. Participants were divided into either the lean group (body mass index $[\mathrm{BMI}]:<23 \mathrm{~kg} / \mathrm{m} 2$ ) or the overweight/obese group (BMI: $\geq 23 \mathrm{~kg} / \mathrm{m} 2)$. Patient demographic, clinical characteristics, metabolic profiles, and laboratory values were collected and compared between groups at baseline and during follow-up for 5 years.At the end of the follow-up, overweight/obese group had a higher risk for developing T2DM than lean group $(11.5 \%$ vs. $0.5 \%, \mathrm{p}<0.001)$. The factors found to be independently associated with increased risk for developing T2DM were $\mathrm{BMI} \geq 23 \mathrm{~kg} / \mathrm{m} 2$ (odds ratio [OR]: 1.075, $\mathrm{p}=0.047$ ). Overweight/obese PCOS patients were found to be at significantly higher risk for developing T2DM than lean PCOS patients. ${ }^{20}$.

S Weerakiet in march 2001 done a case study, To determine the prevalence of abnormalities of glucose metabolism in Asian women with polycystic ovary syndrome (PCOS) and to assess the different impacts of the 1985 and 1999 WHO consultations and the ADA criteria for the diagnosis of type 2 diabetes mellitus (DM). Eighty-five women with PCOS were consecutively included in the study at the Reproductive Endocrinology Unit, Department of Ob-Gyn, Ramathibodi Hospital, Mahidol University. All women underwent a standard oral glucose tolerance test (OGTT). Fasting insulin and testosterone levels were also measured. Seventy-nine women consented to the OGTT. The prevalence of impaired glucose tolerance (IGT) and type 2 DM was 22.8 and $15.2 \%$ with the 1985 WHO criteria, and 20.3 and $17.7 \%$ according to the 1999 WHO consultation criteria, respectively. The recommendation of the ADA using the fasting glucose levels could only determine a prevalence of $6.3 \%$ for type $2 \mathrm{DM}$. The fasting insulin and testosterone levels were significantly higher in DM than IGT and normal glucose tolerance (NGT) subgroups. The PCOS women with abnormalities of glucose metabolism had a greater body mass index (BMI), higher fasting glucose and 2-h post-load glucose levels than those with NGT. The prevalence of glucose intolerance significantly increased with BMI. Similar to other ethnic populations, Asian women with PCOS are at risk of developing IGT and type 2 DM especially if obese. The recommendation of the ADA is not appropriate for the diagnosis of type 2 DM in PCOS women ${ }^{21}$

The study was conducted by Massoud Amini to investigate the prevalence of PCOS in type 2 diabetic patients. Type 2 diabetic women $(n=157)$ of reproductive age were by a convenience sampling method. PCOS was confirmed using the clinical diagnosis criteria. The diabetic patients were divided into two groups according to the presence of PCOS. Baseline demographic characteristics were obtained by questionnaire, and body weight, height, waist circumference, blood pressure and some biochemical indices were measured in both groups. The prevalence of PCOS was high $(8.3 \%, 95 \%$ confidence interval $4.5-13.4 \%)$ in these type 2 diabetic women. The onset of diabetes occurred at a lower age in the PCOS group, who also displayed significantly greater waist circumference and body mass index $(p<0.05)$. No difference in lipid profile, glycosylated hemoglobin or blood pressure was observed between the two group. PCOS is highly prevalent in type 2 diabetic patients. Hence, focusing the treatment on insulin sensitizers in these patients should improve both the metabolic and non-metabolic complications of PCOS. ${ }^{22}$

\section{Conclusion}

It is obvious from the systematic review that the ladies which are obese has a high danger of developing diabetes mellitus type 2 and there is a higher predominance of pcos in those ladies who have type 2 diabetes mellitus instead of non-diabetic and women with pcos have a markedly high danger of developing type 2 diabetes (multiple times more) at that point ladies without pcos.the hazard of type 2 diabetes is especially raised in ladies with pcos in middle-age and accordingly builds up the requirement for routine screening of pcos for diabetes over time.PCOS is related with a two times increased risk for type 2 diabetes $(\mathrm{t} 2 \mathrm{~d})$

\section{References}

1: Stein IF. Amenorrhea associated with bilateral polycystic ovaries. Am J Obstet Gynecol. 1935;29:181-91.

2: Zargar AH, Gupta VK, Wani AI, Masoodi SR, Bashir MI, Laway BA, Ganie MA, Salahuddin M. Prevalence of ultrasonography proved polycystic ovaries in North Indian women with type 2 diabetes mellitus. Reproductive biology and Endocrinology. 2005 Dec;3(1):1-7.

3: Tsilchorozidou T, Overton C, Conway GS. The pathophysiology of polycystic ovary syndrome. Clinical endocrinology. 2004 Jan;60(1):1-7.

4: Zargar AH, Wani AI, Masoodi SR, Laway BA, Bashir MI, Salahuddin M: Epidemiologic and etiologic aspects of hirsutism in Kashmiri women in the Indian Subcontinent. Fertil Steril. 2002, 77: 674-678. 10.1016/S0015- 
0282(01)03241-1.

5: The Rotterdam ESHRE/ASRM - sponsored PCOS Consensus Workshop Group: The Netherlands. Revised 2003 consensus on diagnostic criteria and long term health risks related to polycystic ovary syndrome. Fertil Steril. 2004, 81: 19-25.

6: Clayton RN, Ogden V, Hodgkinson J, Worswick L, Rodin DA, Dyer S, Meade TW: How common are polycystic ovaries in normal women and what is their significance for the fertility of the population?. Clin Endocrinol (Oxf). 1992, 37: 127-134

7: Atiomo WU, Pearson S, Shaw S, Prentice A, Dubbins P: Ultrasound criteria in the diagnosis of polycystic ovary syndrome (PCOS). Ultrasound Med Biol. 2000, 26: 977-980. 10.1016/S0301-5629(00)00219-2.

8: Seli E, Duleba AJ: Treatment of PCOS with metformin and other insulin-sensitizing agents. Curr Diabet Reports. 2004, 4: 69-75.

9: The Expert Committee on the Diagnosis and Classification of Diabetes Mellitus: Follow-up report on the diagnosis of diabetes mellitus. Diabet Care. 2003, 26: 3160-3167.

10: Waterworth DM, Benett ST, Gharani N, McCarthy MI, Hague S, Batty S, Conway GS, White D, Todd JA, Franks S, Williamson R: Linkage and association of insulin gene VNTR regulatory polymorphism with PCOS. Lancet. 1997, 349: 986-990. 10.1016/S0140-6736(96)08368-7.

11: Legro RS, Kunselman A, Dodson WC, Dunaif A: Prevalence and predictors of risk for type 2 diabetes mellitus and impaired glucose tolerance in polycystic ovary syndrome: a prospective, controlled study in 254 affected women. J Clin Endocrinol Metab. 1999, 84: 165-169. 10.1210/jc.84.1.165.

12: Teede HJ, Misso ML, Costello MF, Dokras A, Laven J, Moran L, Piltonen T, Norman RJ, Andersen M, Azziz $\mathrm{R}$ et al. Recommendations from the international evidence-based guideline for the assessment and management of polycystic ovary syndrome. Fertil Steril 2018;110:364-379.

13: (Katrine Hass Rubin, Dorte Glintborg, Mads Nybo, Bo Abrahamsen, Marianne Andersen, Development and Risk Factors of Type 2 Diabetes in a Nationwide Population of Women With Polycystic Ovary Syndrome, The Journal of Clinical Endocrinology \& Metabolism, Volume 102, Issue 10, 1 October 2017, Pages 38483857, https://doi.org/10.1210/jc.2017-01354)

14: A. E. Joham, S. Ranasinha, S. Zoungas, L. Moran, H. J. Teede, Gestational Diabetes and Type 2 Diabetes in Reproductive-Aged Women With Polycystic Ovary Syndrome, The Journal of Clinical Endocrinology \& Metabolism, Volume 99, Issue 3, 1 March 2014, Pages E447-E452, https://doi.org/10.1210/jc.2013-2007

15: (Zargar, A.H., Gupta, V.K., Wani, A.I. et al. Prevalence of ultrasonography proved polycystic ovaries in North Indian women with type 2 diabetes mellitus. Reprod Biol Endocrinol 3, 35 (2005)

16:(Conn, J.J., Jacobs, H.S. and Conway, G.S. (2000), The prevalence of polycystic ovaries in women with type 2 diabetes mellitus. Clinical Endocrinology, 52: 81-86.)

17: Ovalle F, Azziz R. Insulin resistance, polycystic ovary syndrome, and type 2 diabetes mellitus. Fertility and sterility. 2002 Jun 1;77(6):1095-105.

18: Boudreaux, M.Y., Talbott, E.O., Kip, K.E. et al. Risk of T2DM and impaired fasting glucose among pcos subjects: Results of an 8-year follow-up. Curr Diab Rep 6, 77-83 (2006). https://doi.org/10.1007/s11892006-0056-1

19: Peppard HR, Marfori J, Iuorno MJ, Nestler JE. Prevalence of polycystic ovary syndrome among premenopausal women with type 2 diabetes. Diabetes care. 2001 Jun 1;24(6):1050-2.

20: Chantrapanichkul P, Indhavivadhana S, Wongwananuruk T, Techatraisak K, Dangrat C. Prevalence of type 2 diabetes mellitus compared between lean and overweight/obese patients with polycystic ovarian syndrome: a 5-year follow-up study. Archives of gynecology and obstetrics. 2020 Mar;301(3):809-16.

21: Weerakiet S, Srisombut C, Bunnag P, Sangtong S, Chuangsoongnoen N, Rojanasakul A. Prevalence of type 2 diabetes mellitus and impaired glucose tolerance in Asian women with polycystic ovary syndrome. International Journal of Gynecology \& Obstetrics. 2001 Nov 1;75(2):177-84.

22: Massoud Amini, Negar Horri, Mahboubeh Farmani, Sassan Haghighi, Ghoshtasb Sattari, Zahra Pornaghshband \& Ashraf Aminorroaya (2008) Prevalence of polycystic ovary syndrome in reproductiveaged women with type 2 diabetes, Gynecological Endocrinology, 24:8, 423427, DOI: $10.1080 / 09513590802306143$ 